\title{
Graph theoretic techniques in the analysis of uniquely localizable sensor networks
}

\author{
Bill Jackson*and Tibor Jordán ${ }^{\dagger}$
}

Revised version. September 3, 2008

\begin{abstract}
In the network localization problem the goal is to determine the location of all nodes by using only partial information on the pairwise distances (and by computing the exact location of some nodes, called anchors). The network is said to be uniquely localizable if there is a unique set of locations consistent with the given data. Recent results from graph theory and combinatorial rigidity made it possible to characterize uniquely localizable networks in two dimensions. Based on these developments, extensions, related optimization problems, algorithms, and constructions also became tractable. This chapter gives a detailed survey of these new results from the graph theorist's viewpoint.
\end{abstract}

\section{Introduction}

In the network localization problem the locations of some nodes (called anchors) of a network as well as the distances between some pairs of nodes are known, and the goal is to determine the location of all nodes. This is one of the fundamental algorithmic problems in the theory of wireless sensor networks and has been the focus of a number of recent research articles and survey papers, see for example [2, 15, 42, 47].

\footnotetext{
${ }^{*}$ School of Mathematical Sciences, Queen Mary, University of London, Mile End Road, London E1 4NS, England. e-mail: b.jackson@qmul.ac.uk.

${ }^{\dagger}$ Department of Operations Research, Eötvös University, Pázmány Péter sétány 1/C, 1117 Budapest, Hungary. This work was supported by the Mobile Innovation Centre, funded by the Hungarian National Office for Research and Technology. e-mail: jordan@cs.elte.hu.
} 
A natural additional question is whether a solution to the localization problem is unique. The network, with the given locations and distances, is said to be uniquely localizable if there is a unique set of locations consistent with the given data. The unique localizability of a two-dimensional network, whose nodes are 'in generic position', can be characterized by using results from graph rigidity theory. In this case unique localizability depends only on the combinatorial properties of the network: it is determined completely by the distance graph of the network and the set of anchors, or equivalently, by the grounded graph of the network and the number of anchors. The vertices of the distance and grounded graph correspond to the nodes of the network. In both graphs two vertices are connected by an edge if the corresponding distance is explicitly known. In the grounded graph we have additional edges: all pairs of vertices corresponding to anchor nodes are adjacent. The grounded graph represents all known distances, since the distance between two anchors can be obtained from their locations. Before stating the basic observation about unique localizability we need some additional terminology. It is convenient to investigate localization problems with distance information by using frameworks, the central objects of rigidity theory.

A $d$-dimensional framework (also called geometric graph or formation) is a pair $(G, p)$, where $G=(V, E)$ is a graph and $p$ is a map from $V$ to $\mathbb{R}^{d}$. We consider the framework to be a straight line realization of $G$ in $\mathbb{R}^{d}$. Two frameworks $(G, p)$ and $(G, q)$ are equivalent if corresponding edges have the same lengths, that is, if $\|p(u)-p(v)\|=\|q(u)-q(v)\|$ holds for all pairs $u, v$ with $u v \in E$, where $\|$.$\| denotes the Euclidean norm in \mathbb{R}^{d}$. Frameworks $(G, p),(G, q)$ are congruent if $\|p(u)-p(v)\|=\|q(u)-q(v)\|$ holds for all pairs $u, v$ with $u, v \in V$. This is the same as saying that $(G, q)$ can be obtained from $(G, p)$ by an isometry of $\mathbb{R}^{d}$. We shall say that $(G, p)$ is globally rigid, or that $(G, p)$ is a unique realization of $G$, if every framework which is equivalent to $(G, p)$ is congruent to $(G, p)$, see Figure 1.

The next observation shows that the theory of globally rigid frameworks is the mathematical background which is needed to investigate the unique localizability of networks.

Theorem 1.1. [2, 47] Let $N$ be a network in $\mathbb{R}^{d}$ consisting of $m$ anchors located at positions $p_{1}, \ldots, p_{m}$ and $n-m$ ordinary nodes located at $p_{m+1}, \ldots, p_{n}$. Suppose that there are at least $d+1$ anchors in general position. Let $G$ be the grounded graph of $N$ and let $p=\left(p_{1}, \ldots, p_{n}\right)$. Then the network is uniquely localizable if and only if $(G, p)$ is globally rigid.

We shall give a survey of the current status of the theory of globally 

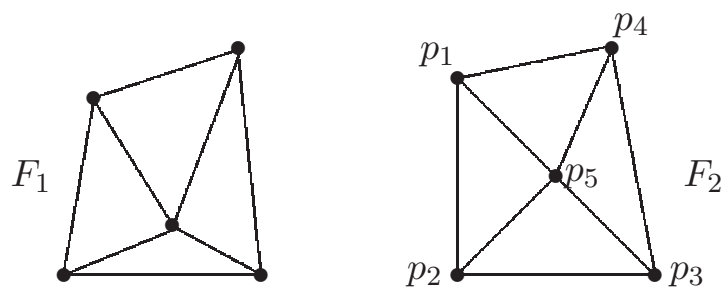

Figure 1: Two realizations of the same graph $G$ in $\mathbb{R}^{2}: F_{1}$ is globally rigid; $F_{2}$ is not since we can obtain a realization of $G$ which is equivalent but not congruent to $F_{2}$ by reflecting $p_{2}$ in the line through $p_{1}, p_{5}, p_{3}$.

rigid graphs and frameworks, focusing on the most relevant cases of twoand three-dimensional frameworks, but stating results for higher dimensions, wherever possible. We will assume that the reader is familiar with the basic terms of graph theory. Readers who are not can find them in the Appendix.

\subsection{Generic frameworks}

It is a hard problem to decide if a given framework is globally rigid. Indeed Saxe [44] has shown that this problem is NP-hard even for 1-dimensional frameworks. Further hardness results can be found in [3], see also [2, 52]. The problem becomes more tractable, however, if we assume that there are no algebraic dependencies between the coordinates of the points of the framework.

A framework $(G, p)$ is said to be generic if the set containing the coordinates of all its points is algebraically independent over the rationals. ${ }^{1}$ Restricting to generic frameworks gives us two important 'stability properties'. The first is that, if $(G, p)$ is a globally rigid $d$-dimensional generic framework then there exists an $\epsilon>0$ such that all frameworks $(G, q)$ which satisfy $\|p(v)-q(v)\|<\epsilon$ for all $v \in V$ are also globally rigid, see for example [10]. The second, which follows from a recent result of Gortler at al. [24], is that if some $d$-dimensional generic realization of a graph $G$ is globally rigid,

\footnotetext{
${ }^{1} \mathrm{~A}$ set $\left\{\alpha_{1}, \alpha_{2}, \ldots, \alpha_{t}\right\}$ of real numbers is algebraically independent over the rationals if, for all non-zero polynomials with rational coefficients $p\left(x_{1}, x_{2}, \ldots, x_{t}\right)$, we have $p\left(\alpha_{1}, \alpha_{2}, \ldots, \alpha_{t}\right) \neq 0$.
} 
then all $d$-dimensional generic realizations of $G$ are globally rigid. We will return to this in Section 11.

\section{Rigidity and global rigidity of graphs}

Rigidity, which is a weaker property of frameworks than global rigidity, plays an important role in the exploration of the structural results of global rigidity as well as in the corresponding algorithmic problems. Intuitively, we can think of a $d$-dimensional framework $(G, p)$ as a collection of bars and joints where vertices correspond to joints and each edge to a rigid bar joining its end-points. The framework is rigid if it has no continuous deformations. Equivalently, and more formally, a framework $(G, p)$ is rigid if there exists an $\epsilon>0$ such that, if $(G, q)$ is equivalent to $(G, p)$ and $\|p(u)-q(u)\|<\epsilon$ for all $v \in V$, then $(G, q)$ is congruent to $(G, p)$.

Rigidity, like global rigidity, is a generic property of frameworks, that is, the rigidity of a generic realization of a graph $G$ depends only on the graph $G$ and not the particular realization. We say that the graph $G$ is rigid, respectively globally rigid or uniquely realizable, in $\mathbb{R}^{d}$ if every (or equivalently, if some) generic realization of $G$ in $\mathbb{R}^{d}$ is rigid, respectively globally rigid.

The problem of characterizing when a graph is rigid in $\mathbb{R}^{d}$ has been solved for $d=1,2$. A graph is rigid in $\mathbb{R}$ if and only if it is connected. The characterization of rigid graphs in $\mathbb{R}^{2}$ is a result of Lovász and Yemini [41], which we will return to in Section 4 . We refer the reader to $[25,50]$ for a detailed survey of the rigidity of $d$-dimensional frameworks.

A similar situation holds for global rigidity: the problem of characterizing when a generic framework is globally rigid in $\mathbb{R}^{d}$ has also been solved for $d=1,2$. A generic framework $(G, p)$ is globally rigid in $\mathbb{R}$ if and only if either $G$ is the complete graph on two vertices or $G$ is 2 -connected. The characterization for $d=2$ uses the following general result of Hendrickson. We say that $G$ is redundantly rigid in $\mathbb{R}^{d}$ if $G-e$ is rigid in $\mathbb{R}^{d}$ for all edges $e$ of $G$.

Theorem 2.1. [26] Let $(G, p)$ be a generic framework in $\mathbb{R}^{d}$. If $(G, p)$ is globally rigid then either $G$ is a complete graph with at most $d+1$ vertices, or $G$ is $(d+1)$-connected and redundantly rigid in $\mathbb{R}^{d}$.

Hendrickson conjectured that the necessary conditions for global rigidity of generic frameworks given in Theorem 2.1 are also sufficient. When $d=1$, this follows from the above mentioned characterizations of rigidity and global 
rigidity. Counterexamples to Hendrickson's conjecture were constructed by Connelly [12] for all $d \geq 3$. The remaining open case, $d=2$, was settled by the following result, which incorporated earlier results from [13, 26] and a new inductive construction for the family of 3-connected redundantly rigid graphs (see Section 5).

Theorem 2.2. [31] Let $(G, p)$ be a 2-dimensional generic framework. Then $(G, p)$ is globally rigid if and only if either $G$ is a complete graph on two or three vertices, or $G$ is 3-connected and redundantly rigid in $\mathbb{R}^{2}$.

Note that the characterizations of globally rigid generic frameworks for $d=1,2$ depend only on the structure of the underlying graph and hence imply the above mentioned result that global rigidity is a generic property in $\mathbb{R}^{d}$, for the special cases when $d=1,2$.

\section{Matroids}

A matroid is an abstract structure which extends the notion of linear independence of vectors in a vector space. We will see that many of the rigidity properties of a generic framework $(G, p)$ are determined by an associated matroid defined on the edge set of $G$. We first need some basic definitions. We refer the reader to the books $[43,45]$ for more information on matroids.

A matroid is an ordered pair $\mathcal{M}=(E, \mathcal{I})$ where $E$ is a finite set, and $\mathcal{I}$ is a family of subsets of $E$, called independent sets, which satisfy the following three axioms.

(M1) $\emptyset \in \mathcal{I}$

(M2) if $I \in \mathcal{I}$ and $D \subseteq I$ then $D \in \mathcal{I}$,

(M3) for all $F \subseteq E$, the maximal independent subsets of $F$ have the same cardinality.

The fundamental example of a matroid is obtained by taking $E$ to be a set of vectors in a vector space and $\mathcal{I}$ to be the family of all linearly independent subsets of $E$.

Given a matroid $\mathcal{M}=(E, \mathcal{I})$, the cardinality of a maximum independent subset of a set $F \subseteq E$ is defined to be the rank of $F$ and denoted by $r(F)$. The rank of $E$ is referred to as the rank of $\mathcal{M}$. A base of $\mathcal{M}$ is a maximum independent subset of $E$. A subset of $E$ which is not independent is said to be dependent. A circuit of $\mathcal{M}$ is a minimal dependent subset of $E$. The matroid $\mathcal{M}$ is said to be connected if every pair of elements of $E$ are contained in a circuit. 
Given a graph $G=(V, E)$, we may define a matroid $\mathcal{M}=(E, \mathcal{I})$ by letting $\mathcal{I}$ be the family of all edge sets of forests in $G$. The rank of a set $F \subseteq E$ is given by $r(F)=|V|-k(F)$, where $k(F)$ denotes the number of connected components in the graph $(V, F)$. A base of $\mathcal{M}$ is the edge set of a forest which has the same number of components as $G$. A circuit of $\mathcal{M}$ is the edge set of a cycle of $G$, and $\mathcal{M}$ is connected if and only if $G$ is 2-connected. This matroid is called the cycle matroid of $G$.

\subsection{Rigidity matrices and matroids}

Let $(G, p)$ be a $d$-dimensional realization of a graph $G=(V, E)$. The rigidity matrix of the framework $(G, p)$ is the matrix $R(G, p)$ of size $|E| \times d|V|$, where, for each edge $e=v_{i} v_{j} \in E$, in the row corresponding to $e$, the entries in the two columns corresponding to vertices $i$ and $j$ contain the $d$ coordinates of $\left(p\left(v_{i}\right)-p\left(v_{j}\right)\right)$ and $\left(p\left(v_{j}\right)-p\left(v_{i}\right)\right)$, respectively, and the remaining entries are zeros. See $[25,50]$ for more details. The rigidity matrix of $(G, p)$ defines the rigidity matroid of $(G, p)$ on the ground set $E$ where a set of edges $F \subseteq E$ is independent if and only if the rows of the rigidity matrix indexed by $F$ are linearly independent. Any two generic $d$-dimensional frameworks $(G, p)$ and $(G, q)$ have the same rigidity matroid. We call this the $d$-dimensional rigidity matroid $\mathcal{R}_{d}(G)$ of the graph $G$. We denote the rank of $\mathcal{R}_{d}(G)$ by $r_{d}(G)$.

As an example, consider a 1-dimensional framework $(G, p)$. In this case, the rows of $R(G, p)$ are just scalar multiples of a directed incidence matrix of $G$. It is well known that a set of rows in this matrix is independent if and only if the corresponding edges induce a forest in $G$. Thus $\mathcal{R}_{1}(G)$ is the cycle matroid of $G$.

Gluck characterized rigid graphs in terms of their rank.

Theorem 3.1. [22] Let $G=(V, E)$ be a graph. Then $G$ is rigid in $\mathbb{R}^{d}$ if and only if either $|V| \leq d+1$ and $G$ is complete, or $|V| \geq d+2$ and $r_{d}(G)=d|V|-\left(\begin{array}{c}d+1 \\ 2\end{array}\right)$.

This characterization does not give rise to a polynomial algorithm for deciding whether a graph is rigid in $\mathbb{R}^{d}$. The problem is that to compute $r_{d}(G)$ we need to determine the rank of the rigidity matrix of a generic realization of $G$ in $\mathbb{R}^{d}$. There is no known polynomial algorithm for calculating the rank of a matrix in which the entries are linear functions of algebraically independent numbers.

We say that a graph $G=(V, E)$ is $M$-independent in $\mathbb{R}^{d}$ if $E$ is independent in $\mathcal{R}_{d}(G)$. Knowing when subgraphs of $G$ are $M$-independent allows 
us to determine the rank of $G$ (and hence determine whether $G$ is rigid), since we can construct a base for $\mathcal{R}_{d}(G)$ by greedily constructing a maximal independent set of $\mathcal{R}_{d}(G)$. This follows from axiom (M3) which guarantees that an independent set which is maximal with respect to inclusion is also an independent set of maximum cardinality. For example, when $d=1$, we have seen that a subgraph is independent if and only if it is a forest. Thus we can determine the rank of $G$ by greedily growing a maximal forest $F$ in $G$. By Theorem 3.1, $G$ is rigid if and only if $F$ has $|V|-1$ edges, i.e. $F$ is a spanning tree of $G$.

\section{The 2-dimensional rigidity matroid}

Sections 4 to 10 of this chapter will mainly be concerned with the case when $d=2$. We will assume that this is the case unless specifically stated otherwise, and suppress the subscript $d$ accordingly.

We first describe the characterization of $M$-independent graphs due to Laman. For $X \subseteq V$ let $i_{G}(X)$ denote the number of edges in $G[X]$, that is, in the subgraph induced by $X$ in $G$.

Theorem 4.1. [39] A graph $G=(V, E)$ is $M$-independent if and only if $i_{G}(X) \leq 2|X|-3$ for all $X \subseteq V$ with $|X| \geq 2$.

The following characterization of rigid graphs due to Lovász and Yemini, which can be deduced from Theorems 3.1 and 4.1, is a slight reformulation of [41, Corollary 4], see [31, Corollary 2.5]. A cover of $G=(V, E)$ is a collection $\mathcal{X}=\left\{X_{1}, X_{2}, \ldots, X_{t}\right\}$ of subsets of $V$ such that $\left\{E\left(X_{1}\right), E\left(X_{2}\right), \ldots, E\left(X_{t}\right)\right\}$ partitions $E$, where $E(X)$ denotes the set of edges in $G[X]$.

Theorem 4.2. [41] Let $G=(V, E)$ be a graph. Then $G$ is rigid if and only if for all covers $\mathcal{X}$ of $G$ we have $\sum_{X \in \mathcal{X}}(2|X|-3) \geq 2|V|-3$.

Theorem 4.2 is illustrated in Figure 2.

A graph $G=(V, E)$ is minimally rigid, or isostatic, if $G$ is rigid, but $G-e$ is not rigid for all $e \in E$. Theorems 3.1 and 4.1 imply that $G$ is minimally rigid if and only if $i_{G}(X) \leq 2|X|-3$ for all $X \subseteq V$ with $|X| \geq 2$ and $|E|=2|V|-3$. Other characterizations for minimally rigid graphs have been given by Lovász and Yemini [41] (for each $e \in E$, the graph obtained from $G$ by adding a new edge parallel to $e$ is the union of two edge disjoint spanning trees), and by Crapo [14] ( $G$ contains three trees such that their edge sets partition $E$, each vertex in $V$ is incident to exactly two of the trees, and the vertex sets of any two non-tivial subtrees that belong to 


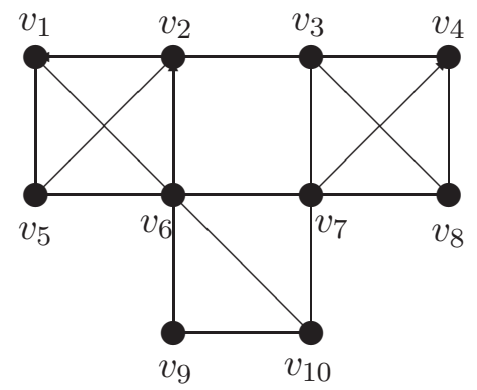

Figure 2: Let $X_{1}=\left\{v_{1}, v_{2}, v_{5}, v_{6}\right\}, X_{2}=\left\{v_{3}, v_{4}, v_{7}, v_{8}\right\}, X_{3}=$ $\left\{v_{6}, v_{7}, v_{9}, v_{10}\right\}, X_{4}=\left\{v_{2}, v_{3}\right\}$, and $\mathcal{X}=\left\{X_{1}, X_{2}, X_{3}, X_{4}\right\}$. Then $\mathcal{X}$ is a cover of $G$. Furthermore $\sum_{X \in \mathcal{X}}(2|X|-3)=16<17=2|V|-3$ so $G$ is not rigid by Theorem 4.2 .

different trees are different.) Note that if $G$ is rigid, then the edge sets of the minimally rigid spanning subgraphs of $G$ form the bases in the rigidity matroid of $G$.

Given a graph $G=(V, E)$, a subgraph $H=(W, C)$ is said to be an $M$-circuit (also called rigidity circuit or generic cycle) in $G$ if $C$ is a circuit (i.e. a minimal dependent set) in $\mathcal{R}(G)$. In particular, $G$ is an $M$-circuit if $E$ is a circuit in $\mathcal{R}(G)$. For example, $K_{4}, K_{3,3}$ plus an edge, and $K_{3,4}$ are all $M$-circuits, see Figure 3. Using Theorem 4.1 we may deduce:

Lemma 4.3. Let $G=(V, E)$ be a graph. The following statements are equivalent.

(a) $G$ is an $M$-circuit.

(b) $|E|=2|V|-2$ and $G-e$ is minimally rigid for all $e \in E$.

(c) $|E|=2|V|-2$ and $i_{G}(X) \leq 2|X|-3$ for all $X \subseteq V$ with $2 \leq|X| \leq|V|-1$.

Recall that a graph $G=(V, E)$ is redundantly rigid if $G-e$ is rigid for all $e \in E$. Equivalently, a graph $G$ is redundantly rigid if and only if $G$ is rigid and each edge of $G$ belongs to an $M$-circuit of $G$. If $G$ is redundantly rigid then $|E| \geq 2|V|-2$ with equality only if $G$ is an $M$-circuit.

We say that a graph $G$ is $M$-connected if $\mathcal{R}(G)$ is connected i.e. every pair of edges of $G$ belongs to an $M$-circuit. We will see that this property has important implications for global rigidity. The graph $K_{3, m}$, for $m \geq 4$, is an example of an $M$-connected graph. It is minimally $M$-connected in the sense that deleting any of its edges results in a graph which is no longer $M$-connected. Note that $K_{3, m}$ is an $M$-circuit only when $m=4$. 

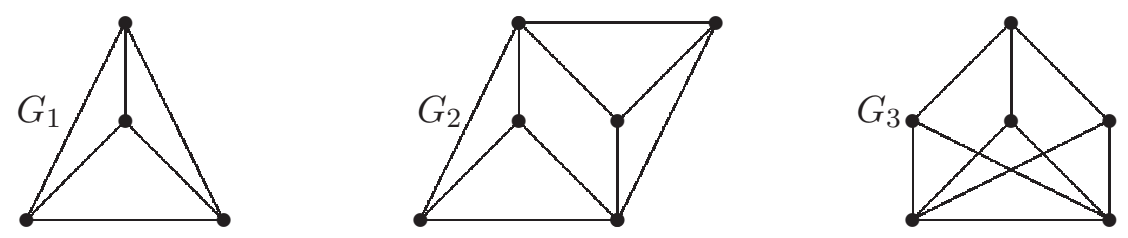

Figure 3: Three examples of $M$-circuits: $G_{1}$ is $K_{4}$ and $G_{3}$ is $K_{3,3}$ plus an edge.

The facts that $M$-circuits are rigid and the union of two rigid graphs with at least two vertices in common is rigid imply that $M$-connected graphs are rigid. Since every edge of an $M$-connected graph belongs to an $M$-circuit, we have:

Lemma 4.4. Every $M$-connected graph is redundantly rigid.

On the other hand, sufficiently connected redundantly rigid graphs are $M$-connected.

Theorem 4.5. [31] Every 3-connected redundantly rigid graph is $M$-connected.

In fact $M$-connected graphs can be characterized as redundantly rigid graphs which have no vertex cut sets of a certain type, see [31, Theorem 3.7]. Note that Theorems 2.2, 4.5, and Lemma 4.4 imply that a graph is globally rigid if and only if it is either a complete graph on at most three vertices or is both 3 -connected and $M$-connected.

\subsection{Decompositions}

We define a rigid component of a graph $G=(V, E)$ to be a maximal rigid subgraph of $G$. It is known (see for example [31, Corollary 2.14]), that any two rigid components of $G$ intersect in at most one vertex and hence that the edge sets of the rigid components of $G$ partition $E$.

It is also known that any two maximal redundantly rigid subgraphs of a graph $G$ can have at most one vertex in common, and hence are edgedisjoint, see [31]. Defining a redundantly rigid component of $G$ to be either a maximal redundantly rigid subgraph of $G$, or a subgraph induced by an 


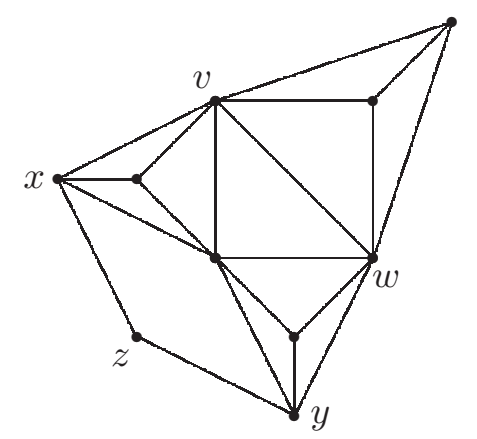

Figure 4: This graph $G$ is rigid so has exactly one rigid component. It has three redundantly rigid components, consisting of $G-z$ and the remaining two copies of $K_{2}$. It has five $M$-connected components: each of the three copies of $K_{4}$, and the remaining two copies of $K_{2}$.

edge which belongs to no $M$-circuit of $G$, we deduce that the redundantly rigid components of $G$ partition $E$. Since each redundantly rigid component is rigid, this partition is a refinement of the partition of $E$ given by the rigid components of $G$.

We may further define an $M$-component of $G$ to be either a maximal $M$ connected subgraph of $G$, or a subgraph induced by an edge which belongs to no $M$-circuit of $G$. We again have the property that any two $M$-connected components of $G$ can have at most one vertex in common, and hence are edge-disjoint, see [31]. Since the $M$-components of $G$ are redundantly rigid by Lemma 4.4, the partition of $E$ given by the $M$-components is a refinement of the partition given by the redundantly rigid components and hence a further refinement of the partition given by the rigid components, see Figure 4.

The partitions of $E$ described above have a stronger matroid property. We say that a matroid $\mathcal{M}=(E, \mathcal{I})$ is the direct sum of two matroids $\mathcal{M}_{1}=$ $\left(E_{1}, \mathcal{I}_{1}\right)$ and $\mathcal{M}_{2}=\left(E_{2}, \mathcal{I}_{2}\right)$ if $E$ is the disjoint union of $E_{1}$ and $E_{2}$ and

$$
\mathcal{I}=\left\{I_{1} \cup I_{2}: I_{1} \in \mathcal{I}_{1}, I_{2} \in \mathcal{I}_{2}\right\} .
$$

The rigidity matroid of a graph $G$ is the direct sum of the rigidity matroids of either the rigid components of $G$, the redundantly rigid components of $G$, 


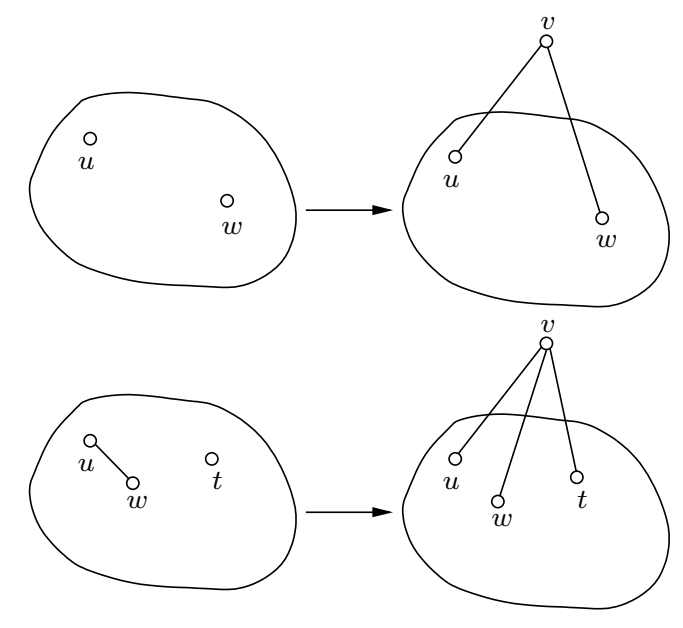

Figure 5: The extension operations.

or the $M$-components of $G$. Furthermore, the vertex sets of the components in each of the above decompositions form a cover of $G$ which minimizes the sum in Theorem 4.2. This minimum value is equal to the rank of $\mathcal{R}_{2}(G)$.

It is an open problem to determine the maximal 'globally rigid components' of vertices of a graph. We will return to this problem in Section 7.

\section{Inductive constructions}

One of the most useful tools in the analysis of (global) rigidity properties of a family of graphs is an inductive construction. In this section we will describe such constructions for rigid graphs and globally rigid graphs.

Let $H$ be a graph. The operation 0-extension (or vertex addition, or Henneberg operation of type $I$ ) adds a new vertex $v$ to $G$ and two edges $v u, v w$ with $u \neq w$. The operation 1-extension (or edge-split, or Henneberg operation of type $I I$ ) subdivides an edge $u w$ of $G$ by a new vertex $v$ and adds a new edge $v z$ for some $z \neq u, w$. An extension is either a 0 -extension or a 1-extension, see Figure 5. The next lemma follows easily from Theorem 4.1.

Lemma 5.1. Let $H$ be a minimally rigid graph and let $G$ be obtained from $H$ by an extension. Then $G$ is minimally rigid.

The following result gives a converse. 
Theorem 5.2. [31] Let $G$ be minimally rigid and let $H$ be a minimally rigid subgraph of $G$. Then $G$ can be obtained from $H$ by a sequence of extensions.

By choosing $H$ to be the subgraph induced by an arbitrary edge of $G$ we obtain the following constructive characterization of minimally rigid graphs (called the Henneberg construction, see [39, 48]).

Theorem 5.3. [48] A graph is minimally rigid if and only if it can be obtained from $K_{2}$ by a sequence of extensions.

As an immediate corollary we deduce that a graph is rigid if and only if it can be obtained from $K_{2}$ be extensions and edge additions.

The analogue of Lemma 5.1 for global rigidity is as follows.

Theorem 5.4. [32] Let $H$ be a globally rigid graph with at least four vertices and let $G$ be obtained from $H$ by a 1-extension. Then $G$ is globally rigid.

A slightly weaker result was previously obtained by Connelly [13], who showed that if $G$ can be obtained from $K_{4}$ by a sequence of 1-extensions then $G$ is globally rigid. His result was a key step in the characterization of globally rigid graphs. The other key ingredient was an inductive construction of 3-connected $M$-connected graphs [31, Theorem 6.15]. Combining both results we obtain

Theorem 5.5. [31] A graph with at least four vertices is globally rigid if and only if it can be obtained from $K_{4}$ by a sequence of 1-extensions and edge additions.

Given a graph $G=(V, E)$, an edge $u v \in E$, and a bipartition $F_{1}, F_{2}$ of the edges incident to $v$ in $G-u v$, the vertex splitting operation on edge uv at vertex $v$ replaces the vertex $v$ by two new vertices $v_{1}$ and $v_{2}$, replaces the edge $u v$ by three new edges $u v_{1}, u v_{2}, v_{1} v_{2}$, and replaces each edge $w v \in F_{i}$ by an edge $w v_{i}, i=1,2$, see Figure 6 . The vertex splitting operation is said to be non-trivial if $F_{1}, F_{2}$ are both non-empty, or equivalently, if each of the split vertices $v_{1}, v_{2}$ has degree at least three.

It is known that vertex splitting preserves rigidity $[49,50]$. Theorem 2.2 can be used to show that it also preserves global rigidity.

Theorem 5.6. [37] Let $H$ be a globally rigid graph and let $G$ be obtained from $H$ by a non-trivial vertex splitting. Then $G$ is also globally rigid.

Inductive constructions can also be used in the problem where a graph $G$ is given and the goal is to construct (a non-generic) globally rigid realization 


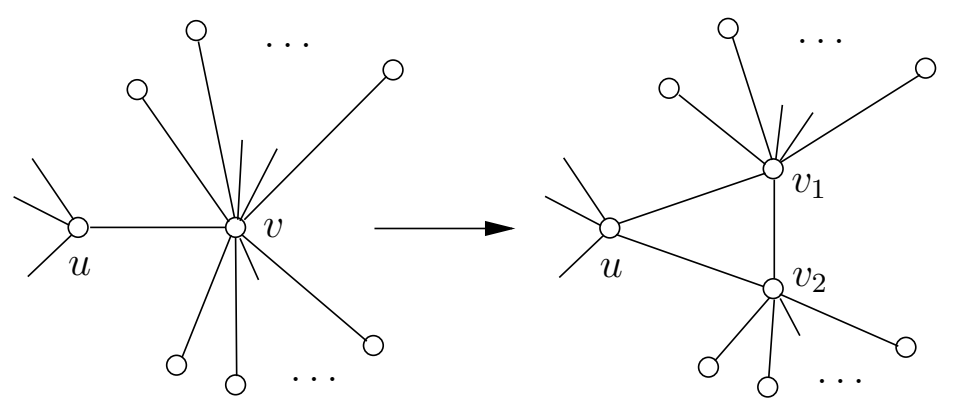

Figure 6: The vertex splitting operation on edge $u v$ and vertex $v$.

of $G$. Given a graph $G=(V, E)$ we say that a 1-extension on the edge $u w$ and vertex $t$ is a triangle-split if $\{u t, w t\} \subseteq E$ (that is, if $u, w, t$ induce a triangle of $G$ ). A graph will be called triangle-reducible if it can be obtained from $K_{4}$ by a sequence of triangle-splits. It is easy to check that triangle-reducible graphs are 3 -connected $M$-circuits. A polynomial time construction for a globally rigid realization of a triangle-reducible graph is given in [37].

The only other known result on the construction of globally rigid realizations is the following. A d-dimensional trilateration ordering of a graph $G=(V, E)$ is an ordering $\left(v_{1}, v_{2}, \ldots, v_{n}\right)$ of $V$ for which the first $d+1$ vertices are pairwise adjacent and at least $d+1$ edges connect each vertex $v_{j}$, $d+2 \leq j \leq n$, to the set of the first $j-1$ vertices. The graph $G$ is a $d$ dimensional trilateration graph if it has a $d$-dimensional trilateration ordering. It is shown in [2] that a $d$-dimensional trilateration graph $G$ is globally rigid in $\mathbb{R}^{d}$, and a construction for a globally rigid realization is given in [15]. Note that 2-dimensional trilateration graphs satisfy $|E| \geq 3|V|-6$, while triangle-reducible graphs are sparser: they have $2|V|-2$ edges, which is the smallest possible number in a globally rigid graph in $\mathbb{R}^{2}$.

\section{Special families of graphs}

\subsection{Graphs of large minimum degree}

We may obtain a sufficient condition for global rigidity in terms of the minimum degree of $G$. The lower bound on the minimum degree in the next theorem is best possible, as shown by two complete graphs of equal size with two vertices in common.

Theorem 6.1. Let $G=(V, E)$ be a graph on $n \geq 4$ vertices with $\delta(G) \geq$ $\frac{n+1}{2}$, where $\delta(G)$ denotes the minimum degree in $G$. Then $G$ is globally rigid. 
We sketch the proof of Theorem 6.1. By Theorem 2.2 it suffices to show that $G$ is 3-connected and redundantly rigid. If $n \leq 4$ then $G$ is complete, so we may suppose that $n \geq 5$. The hypothesis that $\delta(G) \geq(n+1) / 2$ implies that $G$ cannot have a vertex cut of size less than three and hence $G$ is 3-connected. For a contradiction suppose that $H=G-e$ is not rigid for some $e \in E$. Let $C$ be a rigid component of $H$ with as few vertices as possible. Put $D=H-V(C)$. The facts that distinct rigid components of $H$ can share at most one vertex and $\delta(G) \geq \frac{n+1}{2}$, imply that $|V(D)| \geq 4$ and $|V(C)| \leq \frac{n-1}{2}$. Since $C$ is a rigid component of $H$, each vertex of $D$ is adjacent to at most one vertex of $C$ in $H$ by Lemma 5.1. Since $\delta(G) \geq \frac{n+1}{2}$, this implies that $\delta(D) \geq \frac{n-3}{2}$ and all but at most two non-adjacent vertices of $D$ have degree at least $\frac{n-1}{2}$. Hence we may construct a graph $\bar{D}$ with $\delta(\bar{D}) \geq \frac{n-1}{2}$ by adding at most one edge to $D$. Since $|V(C)| \geq 2$, we have $|V(D)| \leq n-2$. We may now use induction on $n$ to deduce that $\bar{D}$ is globally rigid. Since $|V(D)| \geq 4, \bar{D}$ is redundantly rigid and hence $D$ is rigid. Since $|V(C)| \leq \frac{n-1}{2}$ and $\delta(G) \geq \frac{n+1}{2}$, each vertex of $C$ is adjacent to at least one vertex of $D$ in $H$, and all but at most two non-adjacent vertices of $C$ are adjacent to at least two vertices of $D$. We may now use Lemma 5.1 to deduce that $H$ is rigid, a contradiction.

Three-dimensional analogues of Theorem 6.1 are given in [6].

\subsection{Highly connected graphs}

Lovász and Yemini [41] proved that 6-connected graphs are redundantly rigid. Combining this with Theorem 2.2, we may deduce that the same degree of connectivity suffices to give global rigidity.

Theorem 6.2. [31] Let $G$ be 6-connected. Then $G$ is globally rigid.

An infinite family of 5 -connected non-rigid graphs given in [41] shows that the hypothesis on vertex connectivity in both the Lovász-Yemini theorem and Theorem 6.2 cannot be reduced from six to five. On the other hand, Jackson, Servatius and Servatius show in [36] that the connectivity hypothesis can be replaced by a slightly weaker hypothesis of 'essential-6vertex-connectivity' which allows vertex cuts of size four or five as long as they only separate one or at most three vertices, respectively, from the rest of the graph.

We show in [33] that the connectivity hypothesis can be weakened in a more substantial way and still guarantee the rigidity and global rigidity of the graph. To this end we define the following form of mixed vertex and edge connectivity. Let $G=(V, E)$ be a graph. A pair $(U, D)$ with $U \subseteq V$ 
and $D \subseteq E$ is a mixed cut in $G$ if $G-U-D$ is not connected. We say that $G$ is 6 -mixed-connected if $2|U|+|D| \geq 6$ for all mixed cuts $(U, D)$ in $G$. Equivalently, $G$ is 6-mixed-connected if $G$ is 6-edge-connected, $G-v$ is 4 edge-connected for all $v \in V$, and $G-\{u, v\}$ is 2-edge-connected for all pairs $u, v \in V$. It follows that 6 -vertex-connected graphs are 6-mixed-connected and 6-mixed-connected graphs are 3-vertex-connected.

Theorem 6.3. [33] Let $G=(V, E)$ be a 6-mixed-connected graph. Then $G-e$ is globally rigid for all $e \in E$.

The final result of this subsection observes that an even weaker connectivity condition is sufficient to imply that 4-regular graphs are globally rigid. A graph $G=(V, E)$ is said to be cyclically $k$-edge-connected if, for all $X \subseteq V$ such that $G[X]$ and $G[V-X]$ both contain cycles, we have at least $k$ edges from $X$ to $V-X$.

Theorem 6.4. [36] Let $G=(V, E)$ be a cyclically 5-edge-connected 4-regular graph. Then $G$ is globally rigid.

Examples of 4-regular 4-connected graphs and 5-regular 5-connected graphs which are not globally rigid are given in Theorem 6.5 (c),(d) below.

\subsection{Vertex transitive graphs}

Vertex transitive graphs which are rigid or globally rigid were characterized by Jackson, Servatius and Servatius.

Theorem 6.5. [36] Let $G=(V, E)$ be a connected $k$-regular vertex transitive graph on $n$ vertices. Then $G$ is not globally rigid if and only if one of the following holds:

(a) $k=2$ and $n \geq 4$.

(b) $k=3$ and $n \geq 6$.

(c) $k=4$ and $G$ has a 3 -factor $F$ consisting of $s$ disjoint copies of $K_{4}$ where $s \geq 3$.

(d) $k=5$ and $G$ has a 4 -factor $F$ consisting of $s$ disjoint copies of $K_{5}$ where $s \geq 6$.

As a corollary they determine all vertex transitive graphs which are rigid but not globally rigid.

Corollary 6.6. [36] There are exactly four vertex transitive graphs which are rigid but not globally rigid. These are $K_{3,3}$, the triangular prism, the 
graph obtained from $2 C_{4}$ by replacing each vertex by a copy of $K_{4}$ and the graph obtained from $K_{6}$ by replacing each vertex by a copy of $K_{5}$.

(The triangular prism is the 3-regular graph consisting of two disjoint triangles joined by three edges. The graph $2 C_{4}$ is the 4-regular graph obtained from a cycle on four vertices by replacing each edge by two parallel edges.)

\subsection{Random graphs}

We consider three different models of random graphs. Throughout this subsection, we assume that all logarithms are natural.

Our first model is the Erdős-Rényi model of random graphs. Let $G(n, p)$ denote the probability space of all graphs on $n$ vertices in which each pair of vertices is joined by an edge with independent probability $p$, see [7]. A sequence of graph properties $A_{n}$ holds asymptotically almost surely, or a.a.s. for short, in $G(n, p)$ if $\lim _{n \rightarrow \infty} \operatorname{Pr}_{G(n, p)}\left(A_{n}\right)=1$.

Theorem 6.7. [36] Let $G \in G(n, p)$, where $p=(\log n+k \log \log n+w(n)) / n$, and $\lim _{n \rightarrow \infty} w(n)=\infty$.

(a) If $k=2$ then $G$ is a.a.s. rigid.

(b) If $k=3$ then $G$ is a.a.s. globally rigid.

The bounds on $p$ given in Theorem 6.7 are best possible since if $G \in$ $G(n, p)$ and $p=(\log n+k \log \log n+c) / n$ for any constant $c$, then $G$ a.a.s. does not have minimum degree at least $k$, see [7].

Our second model is of random regular graphs. Let $G_{n, d}$ denote the probability space of all $d$-regular graphs on $n$ vertices chosen with the uniform probability distribution. (We refer the reader to [7] for a mathematical procedure for generating the graphs in $G_{n, d}$.) Since globally rigid graphs on at least four vertices are redundantly rigid, the only globally rigid graphs in $G(n, d)$ for $d \leq 3$ are $K_{2}, K_{3}$, and $K_{4}$. The situation changes drastically for $d \geq 4$.

Theorem 6.8. [36] If $G \in G_{n, d}$ and $d \geq 4$ then $G$ is a.a.s. globally rigid.

Our third model is of geometric random graphs. Let Geom $(n, r)$ denote the probability space of all graphs on $n$ vertices in which the vertices are distributed uniformly at random in the unit square and all pairs of vertices of distance at most $r$ are joined by an edge. Suppose $G \in \operatorname{Geom}(n, r)$. Li et al. [40] have shown that if $n \pi r^{2}=\log n+(2 k-3) \log \log n+w(n)$ for $k \geq 2$ a fixed integer and $\lim _{n \rightarrow \infty} w(n)=\infty$, then $G$ is a.a.s. $k$-connected. As noted by Eren et al. [15], this result can be combined with Theorem 6.2 
to deduce that if $n \pi r^{2}=\log n+9 \log \log n+w(n)$ then $G$ is a.a.s. globally rigid. We do not know if this result is best possible. However, it is also shown in [40] that if $n \pi r^{2}=\log n+(k-1) \log \log n+c$ for any constant $c$, then $G$ is not a.a.s. $k$-connected. Thus, if $n \pi r^{2}=\log n+2 \log \log n+c$ for any constant $c$, then $G$ is not a.a.s. 3-connected, and hence is not a.a.s. globally rigid.

\subsection{Unit disk graphs}

A unit disk framework (with radius $R$ ) is a framework $(G, p)$ for which $u v \in E(G)$ if and only if $\|p(u)-p(v)\| \leq R$. A graph $G$ is called a unit disk graph if there is a unit disk realization of $G$. The family of unit disk graphs is a natural model for sensor networks in which the distance between two nodes is known if and only if this distance is at most the sensing radius $R$ of the nodes. It is NP-hard to test whether a graph is a unit disk graph [9], and it is also NP-hard to test whether a unit disk framework is globally rigid [2]. However, it may be possible to use the unit disk property of a unit disk framework $(G, p)$ and bounds on the radius to deduce necessary or sufficient conditions which imply that $(G, p)$ is globally rigid in the sense that it is a unique realization of $G$, with the given edge lengths, as a unit disk framework. This is a largely unexplored area of research.

\subsection{Squares of graphs}

The square $G^{2}$ of a graph $G$ is obtained from $G$ by adding a new edge $u v$ for each pair $u, v \in V(G)$ of distance two in $G$. For example, if we double the sensing radius of a unit disk framework with distance graph $G$, the augmented distance graph will contain its square $G^{2}$. This operation makes a network, whose underlying graph is 2-edge-connected, uniquely localizable.

Theorem 6.9. [1] Let $G$ be a 2-edge-connected graph. Then $G^{2}$ is globally rigid.

\section{Globally linked pairs and uniquely localizable nodes}

Even if a network is not uniquely localizable, the location of some of its vertices, or the distance between some additional pairs of vertices, may be uniquely determined by the distance graph of the network and the set of anchors. This can be modeled as follows. 

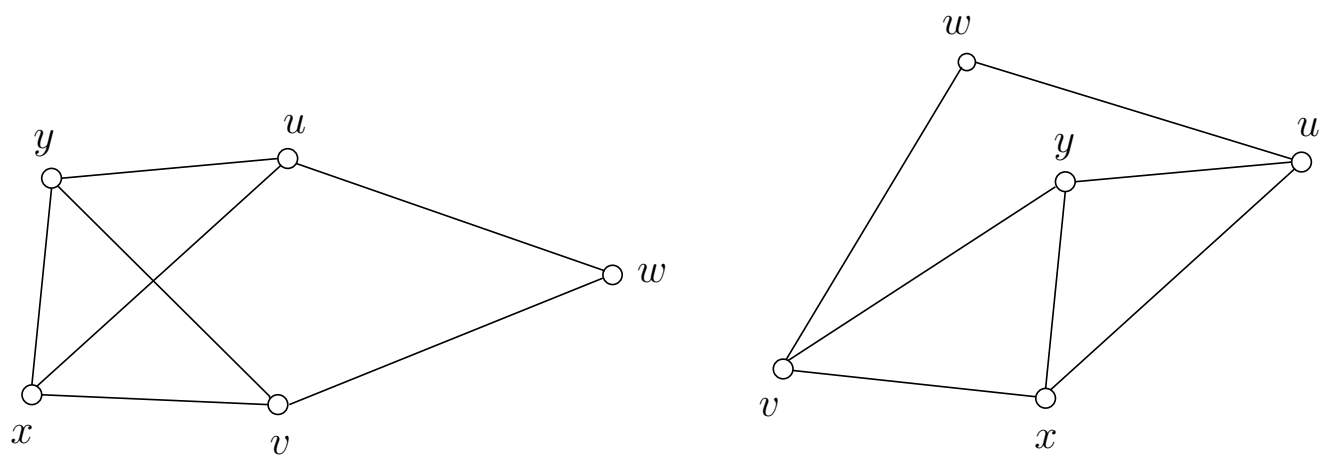

Figure 7: Two equivalent realizations of a rigid graph $G$ which show that the pair $\{u, v\}$ is not globally linked in $G$.

A pair of vertices $\{u, v\}$ in a $d$-dimensional framework $(G, p)$ is globally linked in $(G, p)$ if, in all equivalent frameworks $(G, q)$, we have $\| p(u)-$ $p(v)\|=\| q(u)-q(v) \|$. Thus $(G, p)$ is globally rigid if and only if all pairs of vertices of $G$ are globally linked in $(G, p)$. When $d=1$, it can be seen that a pair of vertices $\{u, v\}$ is globally linked in a generic framework $(G, p)$ if and only if there are two openly disjoint $u v$-paths in $G$. Thus global linkedness is a generic property for 1-dimensional frameworks. Unlike global rigidity, however, 'global linkedness' is not a generic property in $\mathbb{R}^{d}$ when $d \geq 2$. Figures 8 and 7 give an example of a pair of vertices in a rigid graph $G$ which is globally linked in one 2-dimensional generic realization, but not in another: the global linkedness of $\{u, v\}$ depends on the lengths of the edges incident with vertex $w$. We say that a pair of vertices $\{u, v\}$ is globally linked in a graph $G$ in $\mathbb{R}^{d}$ if it is globally linked in all generic $d$-dimensional frameworks $(G, p)$.

We will assume henceforth in this section that $d=2$ and supress specific reference to this value of $d$. We first give a necessary condition for two vertices in a framework to be globally linked. Given two vertices $x, y$ in a graph $G$, let $\kappa_{G}(x, y)$ denote the maximum number of pairwise openly disjoint $x y$-paths in $G$.

Lemma 7.1. [32] Let $(G, p)$ be a generic framework, $x, y \in V(G), x y \notin$ $E(G)$, and suppose that $\kappa_{G}(x, y) \leq 2$. Then $\{x, y\}$ is not globally linked in $(G, p)$.

This necessary condition is also sufficient when $G$ is an $M$-connected graph. 


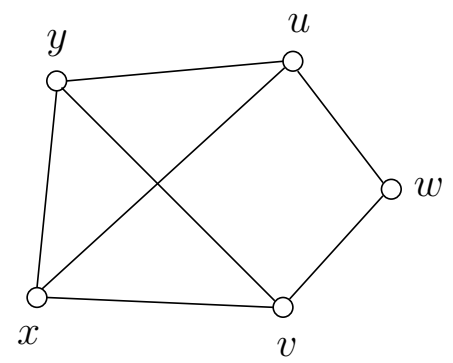

Figure 8: Another realization $(G, p)$ of the rigid graph $G$ of Figure 7. The pair $\{u, v\}$ is globally linked in $(G, p)$ since the lengths of the edges $u w, v w$ preclude the flipping of vertex $v$ about the line containing vertices $x$ and $y$.

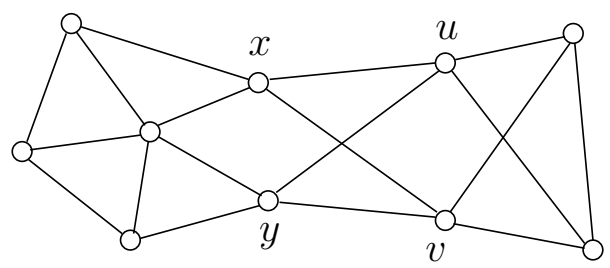

Figure 9: An $M$-connected graph $G$ with three 'cleavage units'. The pairs $\{u, v\}$ and $\{x, y\}$ are globally linked.

Theorem 7.2. [32] Let $G=(V, E)$ be an $M$-connected graph and $x, y \in V$. Then $\{x, y\}$ is globally linked in $G$ if and only if $\kappa_{G}(x, y) \geq 3$.

Theorem 7.2 has the following immediate corollary for graphs which are not necessarily $M$-connected.

Corollary 7.3. [32] Let $G=(V, E)$ be a graph and $x, y \in V$. If either $x y \in E$, or there is an $M$-component $H$ of $G$ with $\{x, y\} \subseteq V(H)$ and $\kappa_{H}(x, y) \geq 3$, then $\{x, y\}$ is globally linked in $G$.

We conjecture that the converse is also true.

Conjecture 7.4. [32] Let $G=(V, E)$ be a graph and $x, y \in V$. Then $\{x, y\}$ is globally linked in $G$ if and only if either $x y \in E$, or there is an $M$-component $H$ of $G$ with $\{x, y\} \subseteq V(H)$ and $\kappa_{H}(x, y) \geq 3$.

Corollary 7.3 can be used to identify large 'globally linked sets of vertices' in a graph $G$. A globally rigid cluster of $G$ is a maximal subset of $V$ in 
which all pairs of vertices are globally linked in $G$. By Corollary 7.3, the vertex sets of the 3 -connected 'cleavage units', (sometimes called 3-connected components or 3-blocks), see [31, Section 3], of the $M$-components of $G$ are globally linked sets in $G$. The truth of Conjecture 7.4 would imply that the vertex sets of these cleavage units (and the pairs of adjacent vertices not included in these units) are precisely the globally rigid clusters of $G$. Note that the vertices of a globally rigid cluster of $G$ need not induce a globally rigid subgraph in $G$. For example, the maximal globally rigid subgraphs of the graph $G$ in Figure 9 are the six copies of $K_{3}$ and the remaining four copies of $K_{2}$. On the other hand, $G$ has three cleavage units, a copy of the wheel on six vertices and two copies of $K_{4}$. The globally rigid clusters of $G$ are precisely the vertex sets of these three cleavage units.

We next consider another generalization of global rigidity, unique localizability, which also has direct applications in sensor network localization, see [23]. Let $(G, p)$ be a generic framework with a designated set $P \subseteq V(G)$ of vertices. We say that a vertex $v \in V(G)$ is uniquely localizable in $(G, p)$ with respect to $P$ if whenever $(G, q)$ is equivalent to $(G, p)$ and $p(b)=q(b)$ for all vertices $b \in P$, then we also have $p(v)=q(v)$. We can think of $P$ as the set of pinned vertices (or anchor nodes in a sensor network). Vertices in $P$ are, by definition, uniquely localizable. It is easy to observe that if $v \in V-P$ is uniquely localizable then $|P| \geq 3$ and there exist three openly disjoint paths from $v$ to $P$ (c.f. Lemma 7.1). As was the case for global linkedness, unique localizablity is not a generic property. Consider the graph given in Figures 8 and 7. If we pin the set $P=\{u, x, y\}$ in the framework of Figure 8, then $v$ is uniquely localizable with respect to $P$. This is not the case if we pin the same set in Figure 7. Thus the unique localizablity of $v$ with respect to $P$ depends on the lengths of the edges incident with $w$.

We say that $v$ is uniquely localizable in the graph $G$ with respect to $P$, if $v$ is uniquely localizable with respect to $P$ in all generic frameworks $(G, p)$. Let $G+K(P)$ denote the graph obtained from $G$ by adding all edges $b b^{\prime}$ for which $b b^{\prime} \notin E$ and $b, b^{\prime} \in P$. The following lemma is easy to prove.

Lemma 7.5. [32] Let $G=(V, E)$ be a graph, $P \subseteq V$ and $v \in V-P$. Then $v$ is uniquely localizable in $G$ with respect to $P$ if and only if $|P| \geq 3$ and $\{v, b\}$ is globally linked in $G+K(P)$ for all (or equivalently, for at least three) vertices $b \in P$.

Lemma 7.5 and Theorem 7.2 imply the following characterization of uniquely localizable vertices when $G+K(P)$ is $M$-connected.

Theorem 7.6. [32] Let $G=(V, E)$ be a graph, $P \subseteq V$ and $v \in V-P$. 
Suppose that $G+K(P)$ is $M$-connected. Then $v$ is uniquely localizable in $G$ with respect to $P$ if and only if $|P| \geq 3$ and $\kappa(v, b) \geq 3$ for all $b \in P$.

Similarly, Lemma 7.5 and Conjecture 7.4 would imply the following characterization of uniquely localizable vertices in an arbitrary graph.

Conjecture 7.7. [32] Let $G=(V, E)$ be a graph, $P \subseteq V$ and $v \in V-P$. Then $v$ is uniquely localizable in $G$ with respect to $P$ if and only if $|P| \geq 3$ and there is an $M$-component $H$ of $G+K(P)$ with $P+v \subseteq V(H)$ and $\kappa_{H}(v, b) \geq 3$ for all $b \in P$.

Theorems 7.2 and 7.6 imply that the sets of globally linked pairs and uniquely localizable vertices can be determined for $M$-connected graphs. Conjectures 7.4 and 7.7 would extend this to all graphs.

We close this section by noting that if $(G, p)$ is a generic realization of an $M$-connected graph $G$, then we can obtain a representative of each distinct congruence class of frameworks which are equivalent to $(G, p)$ by iteratively applying the following operation to $(G, p)$ : choose a 2 -vertex-cut $\{u, v\}$ of $G$ and reflect some, but not all, of the components of $G-\{u, v\}$ in the line through the points $p(u)$ and $p(v)$, see [32, Section 8]. Thus, even though a network with an $M$-connected grounded graph may not be uniquely localizable, all possible sets of locations can be obtained from one set of feasible locations in a simple manner.

\section{Optimal selection of anchors}

Throughout this section, we will again restrict our attention to the 2-dimensional case. Consider the following optimization problem:

Given the set of known distances in a network and a cost function on the nodes, make the network uniquely localizable by designating a minimum cost set of anchor nodes.

Theorems 1.1 and 2.2 imply that, for generic networks, we may reformulate the above problem in the following purely combinatorial form:

Given a graph $G=(V, E)$ and a function $c: V \rightarrow \mathbb{R}_{+}$, find a set $P \subseteq V$, $|P| \geq 3$, for which $G+K(P)$ is globally rigid, and $c(P)=\sum_{v \in P} c(v)$ is minimum.

For example, suppose that $G$ is the graph obtained from a complete graph on four vertices $\{a, b, c, d\}$ by adding two vertices $\{u, v\}$ and two edges $\{d u, u v\}$, and let the cost function be constant. Then an optimal anchor set has cardinality four, and must contain the vertices $\{u, v\}$ as well as two vertices from 
the set $\{a, b, c\}$. Efficient approximation algorithms for solving this problem were obtained in $[19,20]$, using techniques from matroid optimization.

Theorem 8.1. [19, 20] There is a polynomial time $\frac{5}{2}$-approximation algorithm for the problem of finding a minimum cost anchor set which makes a generic framework globally rigid.

\section{Distances and directions}

In this section we consider the unique localizability of sensor networks in which some, or all, of the constraints concern the direction, or bearing, between pairs of nodes rather than the distance. We first look at the simpler case when all constraints are direction constraints.

\subsection{Direction constraints}

Let $(G, p)$ and $(G, q)$ be two $d$-dimensional realizations of a graph $G$. We say that $(G, p)$ and $(G, q)$ are direction equivalent (also called parallel) if $p(u)-p(v)$ is a scalar multiple of $q(u)-q(v)$ for all $u v \in E$. We say that $(G, p)$ and $(G, q)$ are direction congruent if there exists a scalar $\lambda$ and a vector $t$ such that $q(v)=\lambda p(v)+t$ for all $v \in V$. (This is equivalent to saying that $(G, q)$ can be obtained from $(G, p)$ by a translation and dilation.) We call $(G, p)$ globally direction rigid (or tight) if every framework which is direction equivalent to $(G, p)$ is direction congruent to $(G, p)$.

The fact that the direction constraint is a linear constraint enabled Whiteley to characterize globally direction rigid d-dimensional frameworks in terms of the rank of their 'direction rigidity matrix'. He then used this result to obtain a combinatorial characterization for the generic case [50, Theorem 8.2.2]. The following result can be derived from this characterization.

Theorem 9.1. Let $(G, p)$ be a d-dimensional generic framework. Then $(G, p)$ is globally direction rigid if and only if

$$
\sum_{X \in \mathcal{X}}(d|X|-d-1) \geq d|V|-d-1
$$

for all covers $\mathcal{X}$ of $G$.

Note that the characterization of 2-dimensional globally direction rigid generic frameworks given by Theorem 9.1 is identical to the characterization of 2-dimensional rigid generic frameworks given in Theorem 4.2. On the 
other hand, there are three important differences between the cases when all constraints are directions and all constraints are lengths. The direction problem can be solved for all $d$, whereas the length problem has been solved only when $d=1,2$. Furthermore, there is no need to assume that the framework is generic to solve the direction problem and there is no distinction between 'local behaviour' and 'global behaviour' in the direction problem.

Theorem 9.1 implies that a $d$-dimensional generic sensor network in which positions of some anchor nodes and directions between some pairs of nodes are known is uniquely localizable if and only if there are at least two anchor nodes and the underlying 'direction graph' of the network satisfies the condition on covers given in Theorem 9.1. Further results on sensor networks with direction constraints can be found in $[17,38]$.

\subsection{Mixed constraints}

We consider sensor networks in which either distances, directions, or both, are known for some pairs of vertices. The unique localization problem for such networks seems to be at least as hard as that for networks with only distance constraints so we will restrict our attention to the 2-dimensional case (direction constraints are meaningless in one dimension).

A mixed graph is a graph together with a bipartition $D \cup L$ of its edge set. We refer to edges in $D$ as direction edges and edges in $L$ as length edges. A mixed framework $(G, p)$ is a mixed graph $G=(V ; D, L)$ together with a map $p: V \rightarrow \mathbb{R}^{2}$. Two mixed frameworks $(G, p)$ and $(G, q)$ are equivalent if $p(u)-p(v)$ is a scalar multiple of $q(u)-q(v)$ for all $u v \in D$ and $\|p(u)-p(v)\|=\|q(u)-q(v)\|$ for all $u v \in L$. The mixed frameworks $(G, p)$ and $(G, q)$ are congruent if there exists a vector $t \in \mathbb{R}^{2}$ and $\lambda \in\{-1,1\}$ such that $q(v)=\lambda p(v)+t$ for all $v \in V$. This is equivalent to saying that $(G, q)$ can be obtained from $(G, p)$ by a rotation by 0 or 180 degrees and a translation. The mixed framework $(G, p)$ is globally rigid if every framework which is equivalent to $(G, p)$ is congruent to $(G, p)$. It is rigid if there exists an $\epsilon>0$ such that every framework $(G, q)$ which is equivalent to $(G, p)$ and satisfies $\|p(v)-q(v)\|<\epsilon$ for all $v \in V$, is congruent to $(G, p)$.

Servatius and Whiteley [46] developed a rigidity theory for mixed frameworks analogous to that for 'distance constrained' frameworks. One may construct a $|D \cup L| \times 2|V|$ mixed rigidity matrix for a mixed framework $(G, p)$ and use its rows to define the mixed rigidity matroid of $(G, p)$. A generic mixed framework is rigid if and only if its rigidity matrix, or matroid, has rank $2|V|-2$. It follows that the rigidity of mixed frameworks is a generic property and we may define a mixed graph $G$ to be rigid if 

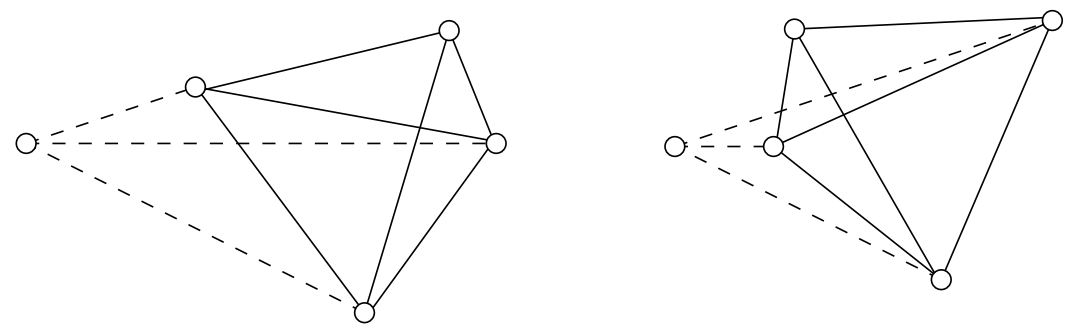

Figure 10: Two equivalent realizations of the same mixed graph. Solid (dashed) edges indicate length (resp. direction) constraints.

every, or equivalently, if some, generic realization of $G$ is rigid. All generic realizations of a mixed graph $G$ have the same mixed rigidity matroid and this matroid is defined to be the mixed rigidity matroid of $G$.

Minimally rigid mixed graphs were characterized by Servatius and Whiteley. For a set $X$ of vertices in a mixed graph $G$ we use $D(X)$ and $L(X)$ to denote the set of direction, resp. length edges in $G[X]$.

Theorem 9.2. [46] Let $G=(V ; D, L)$ be a mixed graph with $|D \cup L|=$ $2|V|-2$. Then $G$ is rigid if and only if for all $X \subseteq V$ with $|X| \geq 2$

$$
i(X) \leq 2|X|-2 \text { when } D(X) \neq \emptyset \neq L(X),
$$

and

$$
i(X) \leq 2|X|-3 \text { otherwise. }
$$

Their result implies the following characterization of rigid mixed graphs.

Theorem 9.3. Let $G=(V ; D, L)$ be a mixed graph. Then $G$ is rigid if and only if for all covers $\mathcal{X}$ of $G$ we have $\sum_{X \in \mathcal{X}} f(X) \geq 2|V|-2$, where $f(X)=2|X|-2$ if $D(X) \neq \emptyset \neq L(X)$, and $f(X)=2|X|-3$ otherwise.

The problem of characterizing when a generic mixed framework $(G, p)$ is globally rigid is still an open problem. We have, however, been able to obtain some partial results. In order to state these in terms of graphs we define a mixed graph $G$ to be globally rigid if all generic realizations of $G$ are globally rigid. ${ }^{2}$

\footnotetext{
${ }^{2}$ It is not known whether global rigidity of mixed frameworks is a generic property. Thus there may exist mixed graphs which have both a globally rigid generic realization and a non-globally rigid generic realization.
} 
We first give a necessary conditions for global rigidity, which is analogous to the '3-connectedness condition' of Theorem 2.2. It uses the following concept. Let $G$ be a 2-connected mixed graph. A 2-separation of $G$ is a pair of subgraphs $G_{1}, G_{2}$ such that $G=G_{1} \cup G_{2},\left|V\left(G_{1}\right) \cap V\left(G_{2}\right)\right|=2$ and $V\left(G_{1}\right)-V\left(G_{2}\right) \neq \emptyset \neq V\left(G_{2}\right)-V\left(G_{1}\right)$. The 2-separation is directionbalanced if both $G_{1}$ and $G_{2}$ contain a direction edge. We say that $G$ is direction balanced if all 2-separations of $G$ are direction balanced.

Theorem 9.4. [34] Every globally rigid mixed graph is 2-connected and direction balanced.

Rigidity is also a necessary condition for global rigidity. Redundant rigidity, however, is no longer necessary. To see this consider a minimally rigid mixed graph $G$ with exactly one length edge $e$. Then $G$ satisfies the hypotheses of Theorem 9.2 , and hence $G-e$ satisfies the hyotheses of Theorem 4.1. Theorem 9.1 now implies that $G-e$ is globally direction rigid, which in turn implies that the mixed graph $G$ is globally rigid. On the other hand, $G-f$ is not rigid for all edges $f$ of $G$.

We next give a result on 1-extensions which is analogous to Theorem 5.4. The operation 1-extension (on edge $u w$ and vertex $z$ ) for a mixed graph $G$ deletes an edge $u w$ and adds a new vertex $v$ and new edges $v u, v w, v z$ for some vertex $z \in V(G)$, with the provisos that at least one of the new edges has the same type as the deleted edge and, if $z=u$, then the two edges from $z$ to $u$ are of different type.

Theorem 9.5. [35] Let $H$ be a globally rigid mixed graph with at least three vertices and let $G$ be obtained from $H$ by a 1-extension on an edge uw. If $H-u w$ is rigid, then $G$ is globally rigid.

In mixed graphs, a special kind of 0 -extension also preserves global rigidity.

Theorem 9.6. [35] Let $G$ and $H$ be mixed graphs with $|V(H)| \geq 2$. Suppose that $G$ can be obtained from $H$ by a 0 -extension which adds a vertex $v$ incident to two direction edges. Then $G$ is globally rigid if and only if $H$ is globally rigid.

Note that if $G$ is obtained by a 0 -extension then $G$ is not redundantly rigid.

We can use Theorems 9.5 and 9.6 to show that a special family of generic mixed frameworks are globally rigid. We say that a mixed graph $G=$ $(V ; D, L)$ is a mixed $M$-circuit if $D \neq \emptyset \neq L$ and $D \cup L$ is a circuit in the mixed rigidity matroid of $G$. Theorem 9.2 can be used to characterize mixed 


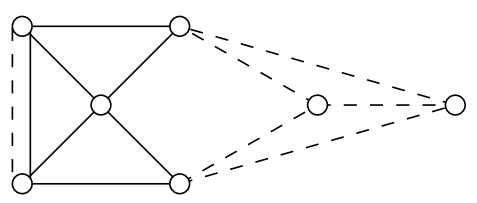

Figure 11: A direction balanced mixed M-circuit.

$M$-circuits and, in particular, show that mixed $M$-circuits are 2-connected. We recently showed that the other necessary condition for global rigidity given in Theorem 9.4 is also sufficient to imply that mixed $M$-circuits are globally rigid.

Theorem 9.7. [34] Let $G$ be a mixed $M$-circuit. Then $G$ is globally rigid if and only if $G$ is direction balanced.

We close this section by noting that we can characterize global rigidity in all dimensions for mixed graphs in which every pair of adjacent vertices is connected by both a length and a direction edge.

Theorem 9.8. [34] Let $G$ be a mixed graph in which every pair of adjacent vertices is connected by both a length and a direction edge, and $(G, p)$ be a generic realization of $G$ in $\mathbb{R}^{d}$. Then $(G, p)$ is globally rigid if and only if $G$ is 2-connected.

\section{Algorithms}

The structural results presented in this chapter give rise to efficient combinatorial algorithms for testing different localizability properties of generic networks and for solving a number of related algorithmic problems in the plane. Here we simply list the basic questions and refer the reader to the references below. The combinatorial characterizations lead us to two kinds of problems. How can we decide whether a given graph $G=(V, E)$ is rigid, redundantly rigid, or $M$-connected? How can we test if $G$ is 3-connected, identify its 'cleavage units', or find its 2-vertex-cuts?

The solution for the questions in the first group boils down to the existence of an efficient subroutine for checking if a set of edges satisfies the count in Theorem 4.1, that is, whether it is $M$-independent in the rigidity matroid (c.f. last paragraph of Section 3). This subroutine can be implemented in $O\left(|V|^{2}\right)$ time by using various alternating path algorithms: methods from matching theory [26], network flows [30], matroid optimization [21], and 
graph orientations $[5,27]$ have been used for this job. By using additional algorithmic techniques, each of these properties can be tested in $O\left(|V|^{2}\right)$ time.

The connectivity related questions of the second group can be solved in $O(|V|+|E|)$ time $[29]$.

\section{Higher dimensional results}

Although most of the known results on globally rigid graphs are concerned with the two dimensional case, some of them extend to higher dimensions, leading to partial results on the unique localizability of 3-dimensional networks. The main tool for working with higher dimensional global rigidity is the 'stress matrix', which was introduced by Connelly [11] and plays a similar role for global rigidity as the rigidity matrix does for rigidity. Indeed, we will see that the global rigidity of a generic framework can be characterized in terms of the rank of a stress matrix in much the same way as Theorem 3.1 characterizes rigidity in terms of the rank of the rigidity matrix. Although the 3-dimensional case is of most practical interest, all the results we know for the 3-dimensional case extend to $d$-dimensions, and we will present them in this context.

Let $G=(V, E)$ be a graph and $(G, p)$ be a realization of $G$ in $\mathbb{R}^{d}$. For each $v \in V$, let $E(v)$ be the set of edges of $G$ which are incident to $v$. An equilibrium stress, or self-stress, for $(G, p)$ is a map $\omega: E \rightarrow \mathbb{R}$ such that, for each $v \in V, \sum_{e=u v \in E(v)} \omega(e)(p(u)-p(v))=0$. We associate a symmetric $|V| \times|V|$ stress matrix $\Omega$ with each equilibrium stress $\omega$ for $(G, p)$ as follows. For each distinct $u, v \in V$, the entry in row $u$ and column $v$ of $\Omega$ is $-\omega(e)$ if $e=u v \in E$ and zero otherwise. The diagonal entries of $\Omega$ are then chosen so that its row and column sums are equal to zero. Connelly [11] proved that the rank of $\Omega$ is at most $|V|-d-1$ and that having a stress matrix with this maximum possible rank is a sufficient condition for the global rigidity of a generic framework.

Theorem 11.1. [13] Let $G=(V, E)$ be a graph and $(G, p)$ be a generic realization of $G$ in $\mathbb{R}^{d}$. If $(G, p)$ has an equilibrium stress $\omega$ such that the associated stress matrix $\Omega$ has rank $|V|-d-1$, then $(G, p)$ is globally rigid.

Connelly conjectured in [13] that this condition is also a necessary condition for the global rigidity of a generic framework $(G, p)$ when $G$ is not complete. His conjecture was recently verified by Gortler, Healy, and Thurston. 
Theorem 11.2. [24] Let $G=(V, E)$ be a graph and $(G, p)$ be a globally rigid generic realization of $G$ in $\mathbb{R}^{d}$. Then either $G$ is a complete graph on at most $d+1$ vertices, or $(G, p)$ has an equilibrium stress $\omega$ such that the associated stress matrix $\Omega$ has rank $|V|-d-1$.

Theorem 11.2 implies that global rigidity in $\mathbb{R}^{d}$ is a generic property. It also gives rise to a randomized algorithm for checking whether a graph is globally rigid in $\mathbb{R}^{d}$, see [24] for more details.

Theorem 11.1 implies the result of Connelly mentioned in Section 5 that if a graph can be obtained from $K_{4}$ by a sequence of 1-extensions, then it is globally rigid in $\mathbb{R}^{2}$. In fact, Connelly used Theorem 11.1 to obtain a $d$-dimensional version of this result. A $d$-dimensional 1-extension of a graph $G$ subdivides an edge $u w$ with a new vertex $v$ and then adds $d-1$ new edges $v z_{i}, 1 \leq i \leq d-1$, with $u \neq z_{i} \neq w$ and $z_{i} \neq z_{j}$ for $1 \leq i<j \leq d-1$. Connelly [13, Theorem 1.5] showed that the property that a $d$-dimensional generic framework has a stress matrix of maximum rank is preserved by a $d$-dimensional 1-extension of the underlying graph. Combining this with Theorem 11.2 we obtain the following $d$-dimensional version of Theorem 5.4.

Theorem 11.3. Let $H$ be a graph with at least $d+2$ vertices and let $G$ be obtained from $H$ by a d-dimensional 1-extension. If $H$ is globally rigid in $\mathbb{R}^{d}$ then $G$ is globally rigid in $\mathbb{R}^{d}$.

As a special case, we obtain Connelly's result [13] that if a graph can be obtained from $K_{d+2}$ by a sequence of $d$-dimensional 1-extensions, then it is globally rigid in $\mathbb{R}^{d}$.

Cheung and Whiteley conjecture that a $d$-dimensional version of the vertex splitting operation also preserves global rigidity in $d$-dimensions. Given a graph $G$, edges $u_{i} v \in E$ for $1 \leq i \leq d-1$, and a bipartition $F_{1}, F_{2}$ of the edges incident to $v$ in $G-\left\{u_{1} v, u_{2} v, \ldots, u_{d-1} v\right\}$, the $d$-dimensional vertex splitting operation replaces the vertex $v$ by two new vertices $v_{1}$ and $v_{2}$ and a new edge $v_{1} v_{2}$, replaces each edge $u_{i} v$ by two new edges $u_{i} v_{1}, u_{i} v_{2}$ for $1 \leq i \leq d-1$, and replaces each edge $w v \in F_{i}$ by an edge $w v_{i}$, for $i=1,2$. The $d$-dimensional vertex splitting operation is said to be non-trivial if $F_{1}, F_{2}$ are both non-empty, or equivalently, if each of the split vertices $v_{1}, v_{2}$ has degree at least $d+1$.

Conjecture 11.4. [10] If $H$ is a globally rigid graph in $\mathbb{R}^{d}$ and $G$ is obtained from $H$ by a non-trivial d-dimensional vertex splitting operation, then $G$ is globally rigid in $\mathbb{R}^{d}$. 
Theorem 5.6 verifies the 2 -dimensional version of Conjecture 11.4. It is open for $d \geq 3$. The preprint [10] contains several additional conjectures on globally rigid graphs in higher dimensions.

\section{References}

[1] B.D.O. Anderson, P.N. Belhumeur, T. Eren, D.K. Goldenberg, A.S. Morse, W. Whiteley, Y.R. YAng, Graphical properties of easily localizable sensor networks, Wireless Netw., 2007.

[2] J. Aspnes, T. Eren, D.K. Goldenberg, A.S. Morse, W. Whiteley, Y.R. Yang, B.D.O. Anderson, P.N. Belhumeur, A theory of network localization, IEEE Trans. on Mobile Computing, vol. 5, issue 12, pp. 1663$1678,2006$.

[3] J. Aspnes, D.K. Goldenberg, and Y.R. Yang, On the computational complexity of sensor network localization, Springer Lecture Notes in Computer Science 3121, Algorithmic Aspects of Wireless Sensor Networks, pp. 32-44, 2004.

[4] A. Berg and T. Jordán, A proof of Connelly's conjecture on 3-connected circuits of the rigidity matroid, J. Combinatorial Theory, Ser. B. Vol. 88, 77-97, 2003.

[5] A. Berg And T. Jordán, Algorithms for graph rigidity and scene analysis, Proc. 11th Annual European Symposium on Algorithms (ESA) 2003, (G. Di Battista, U. Zwick, eds) Springer Lecture Notes in Computer Science 2832, pp. 78-89, 2003.

[6] B. Berger, J. Kleinberg, and T. Leighton, Reconstructing a threedimensional model with arbitrary errors, Journal of the ACM, Vol. 46, No. 2, March 1999, pp. 212-235.

[7] B. Bollobás, Random graphs, Academic Press, New York, 1985.

[8] J.A. Bondy, U.S.R. Murty, Graph theory, Springer, 2008.

[9] H. Breu, D.G. KirkPatrick, Unit disk graph recognition is NP-hard, Comput. Geom. 9 (1998), no. 1-2, 3-24.

[10] M. Cheung, W. Whiteley, Transfer of global rigidity results among dimensions: graph powers and coning, preprint, York University, July 2008.

[11] R. Connelly, Rigidity and energy, Invent. Math., 66(1) (1982), 11-33.

[12] R. Connelly, On generic global rigidity, Applied geometry and discrete mathematics, 147-155, DIMACS Ser. Discrete Math. Theoret. Comput. Sci., 4, Amer. Math. Soc., Providence, RI, 1991.

[13] R. Connelly, Generic global rigidity, Discrete Comput. Geom. 33 (2005) 549-563. 
[14] H. CRAPO, On the generic rigidity of plane frameworks, INRIA research report No. 1278, 1990.

[15] T. Eren, D. Goldenberg, W. Whiteley, Y. R. Yang, A. S. Morse, B. D. O. Anderson, and P. N. Belhumeur, Rigidity, Computation, and Randomization in Network Localization, Proc. of the IEEE INFOCOM Conference, Hong-Kong, March 2004, pp. 2673-2684.

[16] T. Eren, W. Whiteley, A.S. Morse, P.N. Belhumeur, and B.D.O. Anderson, Sensor and network topologies of formations with direction, bearing and angle information between agents, In Proc. of the 42nd IEEE Conference on Decision and Control, pp. 3064-3069, 2003.

[17] T. EREN, Using angle of arrival (bearing) information for localization in robot networks, Turk J Elec Engin, Vol 15, No. 2, 2007, pp. 169-186.

[18] Z. Fekete, Source location with rigidity and tree packing requirements, Operations Research Letters 34, Issue 6, pp. 607-612, 2006.

[19] Z. Fekete, T. Jordán, Uniquely localizable networks with few anchors, Proc. Algosensors 2006, (S. Nikoletseas and J.D.P. Rolim, eds) Springer Lecture Notes in Computer Science 4240, pp. 176-183, 2006.

[20] Z. Fekete, T. Jordán, Algorithms for minimum cost anchor sets in uniquely localizable networks, preprint, 2008.

[21] H.N. Gabow and H.H. Westermann, Forests, frames and games: Algorithms for matroid sums and applications, Algorithmica 7, 465-497 (1992).

[22] H. Gluck, Almost all simply connected closed surfaces are rigid, Geometric topology (Proc. Conf., Park City, Utah, 1974), pp. 225-239. Lecture Notes in Math., Vol. 438, Springer, Berlin, 1975.

[23] D.K. Goldenberg, A. Krishnamurthy, W.C. Maness, Y.R. Yang, A. Young, A.S. Morse, A. Savvides, And B.D.O. Anderson, Network localization in partially localizable networks, Proc. of the IEEE INFOCOM Conference, Miami, March 2005.

[24] S.J. Gortler, A.D. Healy, and D.P. Thurston, Characterizing generic global rigidity, 2007, arXiv:0710.0926v3.

[25] J. Graver, B. Servatius, and H. Servatius, Combinatorial Rigidity, AMS Graduate Studies in Mathematics Vol. 2, 1993.

[26] B. Hendrickson, Conditions for unique graph realizations, SIAM J. Comput. 21 (1992), no. 1, 65-84.

[27] B. HendRICKSON AND D. JACOBS, An algorithm for two-dimensional rigidity percolation: the pebble game, J. Computational Physics 137, 346-365 (1997).

[28] L. Henneberg, Die graphische Statik der starren Systeme, Leipzig, 1911. 
[29] J.E. Hopcroft and R.E. TARJAn, Dividing a graph into triconnected components, SIAM J. Comput. 2 (1973), 135-158.

[30] H. ImaI, On combinatorial structures of line drawings of polyhedra, Discrete Appl. Math. 10, 79 (1985).

[31] B. JaCKSOn And T. Jordán, Connected rigidity matroids and unique realizations of graphs, J. Combinatorial Theory, Ser. B., Vol. 94, 1-29, 2005.

[32] B. JaCkson, T. Jordán, And Z. SzabadKA, Globally linked pairs of vertices in equivalent realizations of graphs, Discrete and Computational Geometry, Vol. 35, 493-512, 2006.

[33] B. Jackson And T. Jordán, A sufficient connectivity condition for generic rigidity in the plane, Discrete Applied Math., in press.

[34] B. JaCKSOn And T. Jordán, Globally rigid circuits of the two-dimensional direction-length rigidity matroid, J. Combinatorial Theory, Ser. B., to appear.

[35] B. JaCKSON AND T. JoRDÁn, Operations preserving global rigidity of generic direction-length frameworks, Egerváry Research Group TR-2008-08, submitted.

[36] B. Jackson, B. Servatius, H. Servatius, The 2-dimensional rigidity of certain families of graphs, J. Graph Theory 54 (2007), no. 2, 154-166.

[37] T. JoRdÁn, Z. SzABADKA, Operations preserving the global rigidity of graphs and frameworks in the plane, 2007, submitted.

[38] B. Katz, M. Gaertler, and D. Wagner, Maximum rigid components as means for direction-based localization in sensor networks, Proc. SOFSEM 2007, LNCS 4362, Jan van Leeuwen et al. (Eds.), pp. 330-341, 2007. Springer, Berlin.

[39] G. Laman, On graphs and rigidity of plane skeletal structures, J. Engineering Math. 4 (1970), 331-340.

[40] X-Y. Li, P-J. Wan, Y. Wang, And C-W. Yi, Fault tolerant deployment and topology control in wireless networks, in Proceedings of the ACM Symposium on Mobile Ad Hoc Networking and Computing (MobiHoc) 2003, Annapolis, MD, June 2003, pp. 117-128.

[41] L. Lovász And Y. Yemini, On generic rigidity in the plane, SIAM J. Algebraic Discrete Methods 3 (1982), no. 1, 91-98.

[42] G. Mao, B. Fidan, B.D.O. Anderson, Localisation, in: Sensor networks and configuration (N.P. Mahalik, ed.), Springer, Berlin, 2007, pp. 281-315.

[43] A. ReCski, Matroid theory and its applications in electric network theory and in statics, Akadémiai Kiadó, Budapest, 1989. 
[44] J.B. SAXE, Embeddability of weighted graphs in $k$-space is strongly NPhard, Tech. Report, Computer Science Department, Carnegie-Mellon University, Pittsburgh, PA, 1979.

[45] A. Schrijver, Combinatorial Optimization, Springer, Berlin, 2003.

[46] B. Servatius And W. Whiteley, Constraining plane configurations in CAD: Combinatorics of directions and lengths, SIAM J. Discrete Math., 12, (1999) $136-153$.

[47] A.M. So And Y. YE, Theory of semidefinite programming for sensor network localization, Math. Program. 109 (2007), no. 2-3, Ser. B, 367-384.

[48] T.S. Tay and W. Whiteley, Generating isostatic frameworks, Structural Topology 11, 1985, pp. 21-69.

[49] W. Whiteley, Vertex splitting in isostatic frameworks, Structural Topology 16, 1990, 23-30.

[50] W. Whiteley, Some matroids from discrete applied geometry. Matroid theory (Seattle, WA, 1995), 171-311, Contemp. Math., 197, Amer. Math. Soc., Providence, RI, 1996.

[51] W. Whiteley, Rigidity and scene analysis, in: Handbook of Discrete and Computational Geometry (J. E. Goodman and J. O'Rourke, eds.), CRC Press, Second Edition, pp. 1327-1354, 2004.

[52] Y. Yemini, Some theoretical aspects of position-location problems, In Proceedings of the 20th Annual IEEE Symposium on Foundations of Computer Science, pages 1-8, 1979.

\section{Appendix}

In what follows we introduce the basic graph theoretical notions that are used in this chapter. For more details see for example [8].

A graph $G=(V, E)$ consists of two sets $V$ and $E$. The elements of $V$ are called vertices (or nodes). The elements of $E$ are called edges. Each edge $e \in E$ joins two vertices from $V$, which are called the endvertices of $e$. The notations $V(G)$ and $E(G)$ are also used for the vertex- and edge-sets of a graph $G$. If vertex $v$ is an endvertex of edge $e$ then $v$ is said to be incident with $e$ and $e$ is incident with $v$. A vertex $v$ is adjacent to vertex $u$ if they are joined by an edge. A graph is simple if the pairs of endvertices of its edges are pairwise distinct. With the exception of the mixed graphs is Section 9, all graphs considered in this chapter will be simple.

The degree of a vertex $v$ in a graph $G$, denoted by $d_{G}(v)$, is the number of edges incident with $v$. A graph is regular if every vertex is of the same degree. It is $k$-regular if every vertex is of degree $k$. 
A path in a graph $G$ from vertex $u$ to vertex $v$ is an alternating sequence of vertices and edges, which starts and ends with $u$ and $v$ (which are its initial and final vertices, respectively), and for which consecutive elements are incident with each other and no internal vertex is repeated. A cycle is a path which contains at least one edge and for which the initial vertex is also the final vertex. A graph is connected if between every pair of vertices there is a path.

A subgraph of a graph $G$ is a graph $H$ with $V(H) \subseteq V(G)$ and $E(H) \subseteq$ $E(G)$. In a graph $G$ the induced subgraph on a set $X$ of vertices, denoted by $G[X]$, has $X$ as its vertex set and it contains every edge of $G$ whose endvertices are in $X$. A subgraph $H$ is a spanning subgraph if $V(H)=V(G)$. A component of a graph $G$ is a maximal connected subgraph. A $k$-factor of a graph $G$ is a $k$-regular spanning subgraph.

The operation of deleting a vertex set $X \subseteq V(G)$ from a graph $G$ removes the vertices in $X$ from $V(G)$ and also removes every edge which has an endvertex in $X$ from $E(G)$. The resulting graph is denoted by $G-X$ (or $G-x$, if $X=\{x\}$ is a single vertex). The operation of deleting an edge set $F \subseteq E(G)$ from a graph $G$ removes the edges in $F$ from $E(G)$. The resulting graph is denoted by $G-F$ (or $G-f$, if $F=\{f\}$ is a single edge).

A forest is a graph without cycles and a tree is a connected forest. A spanning tree of a graph $G$ is a spanning subgraph which is a tree.

A graph is a complete graph if each pair of its vertices is joined by an edge. A complete graph on $n$ vertices is denoted by $K_{n}$. A graph is bipartite if its vertices can be partitioned into two sets in such a way that no edge joins two vertices in the same set. A complete bipartite graph is a bipartite graph in which each vertex in one partite set is adjacent to all vertices in the other partite set. If the two partite sets have cardinalitites $m$ and $n$, then this graph is denoted by $K_{m, n}$. A graph $G$ on $n$ vertices is a wheel, denoted by $W_{n}$, if it has an induced subgraph which is a cycle on $n-1$ vertices and the remaining vertex is joined to all vertices of this cycle.

A $k$-vertex-cut in a graph $G$ is a set $X \subseteq V(G)$ of $k$ vertices for which $G-X$ is not connected. A $k$-edge-cut is a set $F \subseteq E(G)$ of $k$ edges for which $G-F$ is not connected. A graph is called $k$-vertex-connected (or $k$-connected) if it has at least $k+1$ vertices and contains no $l$-vertex-cut for $l \leq k-1$. A graph is $k$-edge-connected if it contains no $l$-edge-cuts for $l \leq k-1$.

Two paths are called openly disjoint if they have no common internal vertex. They are called edge disjoint if they have no common edge. A fundamental theorem of Menger states that if $u$ and $v$ are non-adjacent vertices in graph $G$ then the smallest integer $k$ for which there is a $k$-vertex- 
cut $X$ in $G$ such that $u$ and $v$ are in different components of $G-X$ is equal to the maximum number of pairwise openly disjoint paths from $u$ to $v$. The edge disjoint version of Menger's theorem is as follows. For any pair of vertices $u, v$ in $G$ the smallest integer $k$ for which there is a $k$-edge-cut $F$ in $G$ such that $u$ and $v$ are in different components of $G-F$ is equal to the maximum number of pairwise edge disjoint paths from $u$ to $v$.

An isomorphism between two graphs $G$ and $H$ is a vertex bijection $\phi$ : $V(G) \rightarrow V(H)$ such that $u v \in E(G)$ if and only if $\phi(u) \phi(v) \in E(H)$. A graph automorphism is an isomorphism of the graph to itself. The orbit of a vertex $u$ of a graph $G$ is the set of all vertices $v \in V(G)$ such that there is an automorphism $\phi$ such that $\phi(u)=v$. A graph is vertex-transitive if all the vertices are in the same orbit.

The incidence matrix of a graph $G=(V, E)$ is an $|E| \times|V|$ matrix $I$ where the entry in the row of edge $e$ and vertex $v$ is equal to 1 if $e$ is incident with $v$, and 0 otherwise. The directed incidence matrix of $G$ is obtained from $I$ by replacing exactly one of the two 1's in each row of $I$ by -1 . 